Results Activity Room: Children took similar risks when supervised (.34 \pm .27$)$ and unsupervised $(.30 \pm .24), \mathrm{t}(59)=-1.10$. Bivariate correlations revealed greater risk-taking with more protective/supervision-oriented parents. Multiple linear regression predicting supervised play from PSAPQ scales was significant, $\mathrm{F}(3,53)=4.90, \mathrm{p}<.05, \quad \mathrm{R}^{2}=.22 ; \quad$ only protectiveness significantly predicted, $\mathrm{p}<.05$.

Illustrations Children took fewer risks when supervised (2.29 $\pm 1.55)$ than unsupervised $(1.80 \pm 1.71), \mathrm{t}(59)=2.81, \mathrm{p}<.05$. Bivariate correlations revealed greater risk-taking for more supervision-oriented parents. Multiple linear regression predicting supervised risk-taking from PSAPQ scales was non-significant, $\mathrm{F}(3,54)=2.56, \mathrm{p}=.07, \mathrm{R}^{2}=.13$, although supervision significantly predicted, $\mathrm{p}<.05$.

Conclusion These findings support crucialness of active parental supervision for child injury prevention, and safe riskinvolved exploration for child development. Inattentive supervision may increase risk.

Learning Outcomes Parental supervision quality and type impacts children's risk-taking.

\section{P4.004 CHILD RESTRAINT USE IN SHANGHAI: A MULTI-ROUND CROSS-SECTIONAL OBSERVATIONAL STUDY}

${ }^{1}$ Ting Chen*, ${ }^{2} J$ uanjuan Peng, ${ }^{2}$ Yan Yu, ${ }^{3}$ Abdulgafoor Bachani, ${ }^{3}$ Qingfeng Li. ${ }^{~}$ Department of Population, Family and Reproductive Health, Johns Hopkins Bloomberg School of Public Health, Baltimore, USA; ${ }^{2}$ Shanghai Municipal Center for Disease Control and Prevention, Shanghai, China; ${ }^{3} J o h n s$ Hopkins International Injury Research Unit, Department of International Health, Johns Hopkins Bloomberg School of Public Health, Baltimore, USA

\subsection{6/injuryprev-2021-safety.207}

Background Few studies have assessed the child restraint use in Shanghai. We aim to investigate the prevalence of child restraint use and the potential risk factors affecting child restraint practice in Shanghai.

Methods A cross-sectional observational study was conducted near the pediatric hospitals, kindergartens, entertainment places, and shopping malls between 2015 and 2019 in Shanghai. Trained field workers observed and recoded the child restraint use in all passing motorized vehicles with children passengers at each site. A logistic regression model was used to examine risk factors on non-restraint use among children, adjusting for potential confounders.

Results Eight rounds of data were collected with a total sample size of 12,061 children. The total child restraint use rate was $6.42 \%$. Child restraint use rate rose with fluctuation during the last 5 years, from $5.12 \%$ in round 1 to $8.55 \%$ in round 8 ( $\mathrm{p}$ value $<0.001$ ). Results from the logistic regression model showed that children occupants with these factors had a higher risk for inappropriate child restraint use: children aged 5-12 years compared with those aged younger than 4 years (OR: 2.14; 95\% CI: 1.79-2.55; p<0.001), children traveling with other children occupants compared to those who were the only child in a car (OR: 2.20 ; 95\% CI: $1.51-$ $3.20 ; \mathrm{p}<0.001)$, and children traveling in a taxi compared with those in a sedan/saloon (OR: 32.98; 95\% CI: 16.3766.43; p<0.001).

Conclusion Child restraint use rate was low in Shanghai. Our finding may inform intervention programs among children with those risk factors to promote child restraint use.

\section{P4.005 SUPPORT SYSTEM FOR CHILDREN MOBILITY MANAGEMENT TO IMPROVE SCHOOL TRANSPORT SAFETY}

Dagmara Jankowska-Karpa*. Motor Transport Institute, Warsaw, Poland

10.1136/injuryprev-2021-safety.208

Context Road accidents are main cause of unnatural road deaths among children and youth. Children are vulnerable when they travel unaccompanied to school: walking/cycling, using public transport or dedicated school transport. School transport system requires modernization and more focus on children safety at all stages of the trip.

Process Based on own research regarding effectiveness of Intelligent Transport Systems for school travel, a model of support system for children mobility management was developed to ensure children's safety in a door-to-door perspective. Aim of the system is to i.e. inform parents and school on child location, assist stakeholders supervising school transport to plan optimal routes of buses. The model of the system is composed of five modules: location of children's tags, location of intelligent bus stops, location of children on the bus, location of children at school, optimisation of bus route, safe travelling.

Analysis Different variables were analysed and practical verification of the model was done based on data from one of the regions in Poland. Model of the system refers to all stages of school travel and includes technologies supporting children on way to school.

Outcomes Preliminary validation of the system's model was positive; journey supported by the system was safer than without system. Further analyses should be carried out on a wider scale and model should be expanded with other elements.

Learning Outcomes It is crucial to investigate possibilities of children safety improvements with relation to the use of modern technology measures, which should encompass other road users too.

\section{P4.006 A NOVEL ADOLESCENT INJURY PREVENTION PROGRAM}

${ }^{1}$ Jessica Landry*, ${ }^{2}$ Nimsy Carolina Ramos Velásquez, ${ }^{2}$ María Isabel Bolaños Reyna, ${ }^{1}$ Cinnamon Dixon. 'University of Colorado/Children's Hospital Colorado, Aurora, USA; ${ }^{2}$ Trifinio Center for Human Development, Guatemala

\subsection{6/injuryprev-2021-safety.209}

Background Injury is the leading cause of adolescent mortality in rural Guatemala. Effective injury prevention approaches are conceptualized by local communities to fit the population and environment. Despite the injury burden affecting adolescents worldwide, there is limited engagement of this high-risk population as injury prevention capacity-builders. This project aimed to adapt the World Health Organization (WHO) Child Injury Prevention Course for adolescents, and to assess the acceptability and efficacy of the modified curriculum in increasing injury prevention knowledge and improving selfconfidence in safety behaviors.

Methods The conceptual framework of Adult Learning Theory was used to modify the WHO course into an engaging curriculum for adolescents. The curriculum was then translated into Spanish and implemented with a class of 11 adolescents 